\title{
Doenças sexualmente transmissíveis atendidas em unidade primária de saúde no Nordeste do Brasil
}

\author{
Sexually transmitted diseases in primary health care \\ unit in Northeastern Brazil
}

\author{
Maria Alix Leite Araújo ${ }^{1}$, Ana Fátima Braga Rocha ${ }^{1}$, \\ Elani Graça Ferreira Cavalcante ${ }^{2}$, Heber José de Moura ${ }^{3}$, \\ Marli Teresinha Gimeniz Galvão ${ }^{4}$, Ana Cristina Martins Uchoa Lopes ${ }^{1}$
}

\begin{abstract}
Resumo
Objetivou-se analisar os casos de doenças sexualmente transmissíveis (DST) atendidos em uma unidade primária de saúde em Fortaleza, Ceará, estudo retrospectivo que analisou 5.590 prontuários de casos de DST atendidos nos anos de 1999 a 2009. Os dados foram coletados de março a junho de 2010 e analisados pelo SPSS 18.0. Pessoas jovens com boa escolaridade e que tiveram múltiplos parceiros sexuais nos últimos três meses apresentaram mais úlcera e verruga. Encontrou-se associação entre apresentar verruga e ter resultado reagente para o HIV e apresentar úlcera e ter resultado reagente de VDRL. Quando analisados por sexo, as mulheres apresentaram mais úlcera, verruga, mais de uma síndrome genital no momento da consulta e aceitaram mais realizar os exames de VDRL e HIV. Já os homens tiveram mais resultado reagente para o HIV. Homens e mulheres com DST apresentaram especificidades comportamentais que os tornam mais vulneráveis ao HIV e sífilis.
\end{abstract}

Palavras-chave: atenção primária à saúde; doenças sexualmente transmissíveis; prevenção de doenças transmissíveis.

\section{Abstract}

This study aimed to assess cases of sexually transmitted diseases (STDs) in a primary healthcare unit in the city of Fortaleza, Ceará. A retrospective study that analyzed 5,590 medical records of STD cases treated between 1999 and 2009. Data were collected from March to June 2010 and analyzed using SPSS 18.0. Young people with high education levels and those with multiple sex partners in the past three months presented with more ulcers and warts. The presence of warts was associated to reactive HIV result, and the presence of ulcers was associated to reactive VDRL result. As for gender, women presented more ulcers, warts, and more than one genital syndrome at physical examination and were more willing to perform VDRL and HIV testing. Men and women with STDs presented behavioral specificities that make them more vulnerable to HIV and syphilis.

Keywords: primary health care; sexually transmitted diseases; infectious diseases prevention.

${ }^{1}$ Programa de Pós-graduação em Saúde Coletiva, Universidade de Fortaleza (UNIFOR) - Fortaleza (CE), Brasil.

${ }^{2}$ Centro de Saúde Meireles, Secretaria de Saúde do Estado do Ceará - Fortaleza (CE), Brasil.

${ }_{3}^{3}$ Programa de Pós-graduação em Administração (PPGA), Universidade de Fortaleza (UNIFOR) - Fortaleza (CE), Brasil.

${ }^{4}$ Programa de Pós-graduação em Enfermagem, Universidade Federal do Ceará (UFC) - Fortaleza (CE), Brasil.

Trabalho realizado na Universidade de Fortaleza (UNIFOR) - Fortaleza (CE), Brasil.

Endereço para correspondência: Maria Alix Leite Araujo - Av. Washington Soares, Bloco S - 01, 1321 - Edson Queiroz - CEP: 60811-905 - Fortaleza (CE), Brasil

-Email: alix.araujo@hotmail.com

Fonte de financiamento: nenhuma.

Conflito de interesses: nada a declarar. 


\section{INTRODUÇÃO}

A prevalência de doenças sexualmente transmissíveis (DST) continua crescendo em todo o mundo, com estimativa de que ocorram no mundo, a cada ano, 448 milhões de novos casos $^{1}$ e, desses, 10 a 12 milhões somente no Brasil ${ }^{2}$. No ano de 2005, a estimativa é de que ocorreram, respectivamente no Sudeste da Ásia e no Pacífico Ocidental, aproximadamente 7,39 e 32,69 milhões de casos de clamídia; 8,37 e 9,43 milhões de casos de gonorreia; 11,77 e 2,54 milhões de casos de sífilis; e 26,91 e 25,76 milhões de casos de tricomoníase. Essas são as regiões que têm o maior número de DST curáveis dentre todas as regiões do planeta ${ }^{3}$.

Anualmente, nos Estados Unidos, ocorrem aproximadamente 19 milhões de novos casos, acometendo especialmente adolescentes e adultos jovens, com custos que variam de 10 a 17 bilhões de dólares ${ }^{4}$. Esses dados podem ser subestimados, considerando que há falta de regularidade nos registros das DST, mesmo as de notificação obrigatória, o que torna os dados escassos e inconclusivos, impossibilitando o desenho de estratégias preventivas eficazes, ou seja, adaptadas às reais necessidades $\mathrm{e}$ ao perfil da população.

As DST podem causar infertilidade ${ }^{5}$, câncer cervical ${ }^{6}$, anal ${ }^{7}$ e de pênis ${ }^{8}$. Ademais, podem aumentar o risco de infecção pelo HIV $^{9}$. Estudo realizado com homens e mulheres que procuraram clínicas de DST em seis capitais brasileiras encontrou prevalência de $51 \%$, sendo $14,4 \%$ para as doenças bacterianas e $41,9 \%$ para as virais ${ }^{10}$. Outros estudos mostram que essas infecções se diferenciam segundo o tipo de patologia e a região estudada ${ }^{10-12}$.

O tratamento das DST deve ser realizado em unidades de atenção primária, por meio da abordagem sindrômica, estratégia recomendada pela Organização Mundial da Saúde (OMS) e pelo Ministério da Saúde (MS) do Brasil². Essa estratégia prevê o diagnóstico precoce e o tratamento imediato das pessoas acometidas, evitando perdas no seguimento, prevenindo sequelas e proporcionando a quebra imediata da cadeia de transmissão. Permite a realização do atendimento mesmo em locais onde há escassez de laboratórios, situação que ocorre normalmente nos países em desenvolvimento ${ }^{13}$.

Estudo que enviou um questionário aos diretores dos programas nacionais de DST de 19 países da América Latina e do Caribe e que teve por objetivo avaliar as informações epidemiológicas e os aspectos organizacionais dos programas de DST mostrou que em todos os países envolvidos o atendimento das DST encontrava-se inserido nos serviços de atenção primária ${ }^{14}$. Ocorre que como a coleta de dados ocorreu via e-mail, é muito provável que esses diretores, por estarem distantes da realidade desses serviços, desconheçam efetivamente a realidade operacional do atendimento das DST nesse nível de atenção.
No Brasil, as unidades primárias de saúde ainda não incorporaram o atendimento às DST na rotina dos serviços, apesar do protocolo estabelecido para aplicação da abordagem sindrômica. Muitos portadores, especialmente os homens, referem dificuldade de acesso ao tratamento ${ }^{15}$, por esse motivo preferem procurar as farmácias privadas, pois não precisam esperar dias por atendimento e nem enfrentar longas filas ${ }^{16}$.

Os profissionais também não se sentem preparados para abordar a temática das $\mathrm{DST}^{17}$, deixando lacunas importantes em relação à prevenção $\mathrm{e}$ ao aconselhamento, especialmente quando se trata da convocação e tratamento dos parceiros sexuais ${ }^{18}$. Por outro lado, não existe uma proposta de educação permanente desses profissionais e nem reflexão acerca das estratégias de superação das dificuldades de inserção do atendimento das DST na rotina dos serviços, já tão sobrecarregados por outras atividades.

Para Gaydos, as áreas fundamentais para compreender e verificar sua relação com as DST e o seu controle incluem: epidemiologia, comportamento, diagnóstico, tratamento, vacinação, educação dos pacientes e educação de prestadores de serviços. ${ }^{4}$.

Destaca-se, portanto, a importância de estudos epidemiológicos que analisem os casos de DST atendidos pela abordagem sindrômica, considerando que ao longo dos anos ocorreram mudanças significativas nas práticas sexuais e no perfil da população acometida, provocando incremento no número de casos. Conhecer os aspectos envolvidos nos casos atendidos com síndromes genitais é indispensável para o desenho de estratégias de prevenção. Por esse motivo, este estudo tem por objetivo analisar os casos de DST atendidos em uma unidade primária de saúde em Fortaleza, Ceará.

\section{MÉTODO}

Estudo retrospectivo que analisou os casos de síndromes genitais atendidos pela abordagem sindrômica em uma unidade primária de saúde no município de Fortaleza, Ceará. Essa unidade se localiza em área nobre da cidade e próxima à região de grande atração turística. Atende uma média mensal de 200 pacientes, seguindo o fluxograma proposto pela $\mathrm{OMS}^{19}$ e pelo $\mathrm{MS}^{2}$. Todos os pacientes têm dois retornos agendados: um com sete dias, para avaliação do tratamento e entrega do exame VDRL, e outro com 30 dias, para entrega do anti-HIV. A notificação dos parceiros obedece às recomendações do MS definidas para cada síndrome ${ }^{2}$.

Nessa unidade, o atendimento de DST foi implantado por meio de um projeto iniciado em meados da década de 1990 que teve aproximadamente seis anos de duração. Contou com financiamento da Universidade de Bordeaux, França, do MS do Brasil, da Secretaria de Saúde do Estado do Ceará e do Município de Fortaleza. Esse projeto contribuiu com a 
aquisição de medicamentos, preservativos e no treinamento dos profissionais.

Participaram do estudo todas as pessoas com DST atendidas pela abordagem sindrômica. Consideraram-se casos de DST todos aqueles que tinham diagnóstico sindrômico de úlceras genitais, verrugas genitais, corrimento vaginal, corrimento uretral e/ou dor pélvica confirmados pelo profissional de saúde durante o exame físico e/ou ginecológico. Foram incluídos os casos que apresentaram uma ou mais síndromes genitais.

Na ocasião do atendimento e após a obtenção de autorização escrita, são solicitados os exames de Veneral Desease Research Laboratory (VDRL) e anti-HIV a todos os pacientes, com aconselhamento pré e pós-teste. Esses exames são colhidos logo após o atendimento, sendo o VDRL realizado no próprio laboratório da unidade, com resultado disponível em média sete dias após a coleta, e a amostra sorológica do anti-HIV enviada ao Laboratório Central do Estado do Ceará (LACEN-CE), com retorno do resultado em, na média, 30 dias.

A coleta de dados foi realizada no período de março a junho de 2010 nas fichas de atendimento e nos prontuários médicos dos pacientes com DST atendidos durante os anos de 1999 a 2009.

Foram analisadas as variáveis sociodemográficas (sexo, idade, escolaridade em anos de estudo completos, procedência); comportamentais (número de parceiros sexuais nos últimos três meses, retorno à consulta para reavaliação); realização e resultados dos exames de VDRL e anti-HIV; síndrome genital (úlceras e/ou verrugas genitais, corrimento vaginal, corrimento uretral e/ou dor pélvica). Considerou-se que retornaram à consulta somente aqueles que vieram à unidade no prazo máximo de 90 dias após o primeiro atendimento, tempo considerado para encerramento do caso. Já nos casos de VDRL e anti-HIV, consideraram-se aqueles cujos resultados constavam na ficha de atendimento ou no prontuário.

Os dados foram processados usando-se o pacote estatístico Statistical Package for the Social Sciences (SPSS) versão 18.0. Também se obtiveram resultados univariados para cada uma das variáveis adotadas, como forma de caracterizar melhor o contexto da análise. Além disso, testes de associação entre variáveis, usando a estatística $\chi^{2}$, foram realizados, utilizando-se o nível de significância de $5 \%$.

Esta pesquisa fez parte de um estudo mais amplo sobre abordagem sindrômica das DST aprovada pelo Comitê de Ética em Pesquisa da Escola de Saúde Pública do Estado do Ceará, conforme protocolo n. 126/2008, sendo garantido o sigilo e confidenciabilidade das informações.

\section{RESULTADOS}

Foram analisados 5.590 prontuários e fichas de atendimento de pessoas com DST. Na Tabela 1, em que estão descritos os dados sociodemográficos dos indivíduos, observa-se que
$4.227(75,6 \%)$ eram do sexo feminino, 2.627 (47,0\%) tinham idade entre 20 e 29 anos, 2.131 (38,1\%) tinham de 5 a 8 anos completos de estudo e que $5.434(97,2 \%)$ eram da cidade de Fortaleza.

Realizaram os exames anti-HIV e VDRL 4.436 (79,4\%) e $4.889(87,5 \%)$ pessoas, respectivamente. Em relação ao anti-HIV, $973(22,1 \%)$ eram homens e 3.439 (77,9\%), mulheres, e quanto ao VDRL, 1.363 (24,4\%) eram homens e 4.227 (75,6\%) eram mulheres. A proporção de resultados reagentes de anti-HIV foi de $0,53 \%$ (30/5.590) para os homens e de 0,46\% (26/5.590) para as mulheres. Do total de atendimentos, retornaram para consulta 4.621 (94,3\%) pacientes (dados não apresentados em tabelas).

A Tabela 2 apresenta uma análise das úlceras e verrugas para as variáveis idade, escolaridade, número de parceiros sexuais e resultado dos exames anti-HIV e VDRL. Encontrou-se associação estatisticamente significativa para apresentar úlcera: escolaridade maior ou igual a nove anos completos de estudo $(\mathrm{p}=0,025)$, ter se relacionado com dois ou mais parceiros sexuais nos últimos três meses $(\mathrm{p}<0,001)$ e resultado reagente para o VDRL ( $<<0,001)$; para apresentar verruga: idade igual ou inferior a 19 anos $(\mathrm{p}<0,001)$, ter nove ou mais anos completos de estudo ( $\mathrm{p}<0,001)$ e resultado reagente para o HIV $(\mathrm{p}=0,001)$.

$\mathrm{Na}$ Tabela 3 está apresentada uma análise por sexo do número de síndromes genitais, presença de úlcera e verruga, realização e resultado dos exames de VDRL e anti-HIV. As mulheres apresentaram mais síndromes genitais $(\mathrm{p}<0,001)$, úlcera genital $(\mathrm{p}<0,001)$, verruga genital $(\mathrm{p}<0,001)$ e aceitaram realizar mais os exames de VDRL ( $\mathrm{p}<0,001)$ e anti-HIV ( $\mathrm{p}<0,001)$. Os homens tiveram mais resultados reagentes para o HIV $(\mathrm{p}<0,001)$.

Tabela 1. Variáveis sociodemográficas dos indivíduos atendidos com queixas genitais, Fortaleza, Ceará, 1999 a 2009 ( $\mathrm{N}=5.590)$

\begin{tabular}{lcc}
\multicolumn{1}{c}{ Variáveis } & $\mathbf{N}$ & $\%$ \\
dade & & \\
$\leq 19$ & 930 & 16,6 \\
$20-29$ & 2627 & 47,0 \\
$\geq 30$ & 2033 & 36,4 \\
Sexo & & \\
$\quad$ Masculino & 1363 & 24,4 \\
Feminino & 4227 & 75,6 \\
Escolaridade & & \\
$0-4$ & 1471 & 26,3 \\
$5-8$ & 2131 & 38,1 \\
$\geq 9$ & 1939 & 34,7 \\
Ignorado & 49 & 0,9 \\
Procedência & & \\
Fortaleza & 5434 & 97,2 \\
Outro município & 156 & 2,8
\end{tabular}


Tabela 2. Presença de úlcera e verruga por idade, escolaridade, número de parceiros sexuais e resultado dos exames anti-HIV e VDRL, Fortaleza, Ceará, 1999 a 2009

\begin{tabular}{|c|c|c|c|c|c|c|c|c|c|c|}
\hline \multirow{3}{*}{$\begin{array}{l}\text { Variáveis } \\
\text { Idade }(\mathrm{n}=5590)\end{array}$} & \multicolumn{4}{|c|}{ Úlcera* $^{*}$} & \multirow{3}{*}{$\begin{array}{r}\text { Valor p } \\
0,229\end{array}$} & \multicolumn{4}{|c|}{ Verruga $^{*}$} & \multirow{3}{*}{$\begin{array}{l}\text { Valor } \mathbf{p} \\
<0,001\end{array}$} \\
\hline & \multicolumn{2}{|c|}{ Sim } & \multicolumn{2}{|c|}{ Não } & & \multicolumn{2}{|c|}{ Sim } & \multicolumn{2}{|c|}{ Não } & \\
\hline & $\mathrm{n}$ & $\%$ & $\mathrm{~N}$ & $\%$ & & $\mathrm{n}$ & $\%$ & $\mathrm{n}$ & $\%$ & \\
\hline$\leq 19$ & 64 & 6,9 & 866 & 93,1 & & 514 & 55,3 & 416 & 44,7 & \\
\hline $20-29$ & 141 & 5,4 & 2486 & 94,6 & & 1300 & 49,5 & 1327 & 50,5 & \\
\hline$\geq 30$ & 121 & 6,0 & 1912 & 94,0 & & 689 & 33,9 & 1344 & 66,1 & \\
\hline Escolaridade $(n=5541)$ & & & & & 0,025 & & & & & $<0,001$ \\
\hline $0-4$ & 71 & 4,8 & 1400 & 95,2 & & 476 & 32,4 & 995 & 67,6 & \\
\hline $5-8$ & 119 & 5,6 & 2012 & 94,4 & & 936 & 43,9 & 1195 & 56,1 & \\
\hline$\geq 9$ & 135 & 7,0 & 1804 & 93,0 & & 1068 & 55,1 & 871 & 44,9 & \\
\hline No de parceiros $(\mathrm{n}=5499)$ & & & & & $<0,001$ & & & & & 0,327 \\
\hline Sem parceiro & 21 & 5,4 & 367 & 94,6 & & 173 & 44,6 & 215 & 55,4 & \\
\hline 1 & 224 & 5,1 & 4209 & 94,9 & & 1963 & 44,3 & 2470 & 55,7 & \\
\hline$\geq 2$ & 70 & 10,3 & 608 & 89,7 & & 321 & 47,3 & 357 & 52,7 & \\
\hline Resultado VDRL $(\mathrm{n}=4900)$ & & & & & $<0,001$ & & & & & 0,692 \\
\hline Reagente & 74 & 18,4 & 328 & 81,6 & & 106 & 26,4 & 296 & 73,6 & \\
\hline Não reagente & 214 & 4,8 & 4284 & 95,2 & & 2093 & 46,5 & 2405 & 53,5 & \\
\hline Resultado anti-HIV ( $\mathrm{n}=4436)$ & & & & & 0,259 & & & & & 0,001 \\
\hline Reagente & 5 & 8,9 & 51 & 91,1 & & 38 & 67,9 & 18 & 32,1 & \\
\hline Não reagente & 238 & 5,5 & 4118 & 94,5 & & 1944 & 44,6 & 2412 & 55,4 & \\
\hline
\end{tabular}

*Síndrome comum aos sexos

Tabela 3. Análise, por sexo, do número de síndromes genitais, presença de úlcera e verruga, realização e resultado dos exames de VDRL e anti-HIV, Fortaleza, Ceará, 1999 a 2009

\section{Variáveis}

No de síndromes $(\mathrm{n}=5.590)$

1

$>1$

Úlcera genital ${ }^{\star}(\mathrm{n}=5.590)$

Sim

Não

Verruga genital $^{\star}(n=5.590)$

Sim

Não

Realizou VDRL ( $\mathrm{n}=5.590)$

Sim

Não/Ignorado

Resultado VDRL ( $\mathrm{n}=4.889$ )

Reagente

Não regente

Realizou anti-HIV ( $\mathrm{n}=5.590)$

Sim

Não/Ignorado

Resultado anti-HIV $(\mathrm{n}=4.436)$

Reagente

Não reagente

\section{Masculino $(\mathrm{n}=1.363)$}

1326

37

149

1214

877

486

1152

211

102

1050

987

376

30

943
Sexo

Feminino $(n=4.227)$

Valor $\mathrm{p}$

$<0,001$

3822

405

74,2

91,6

177

4050

$<0,001$

1626

2601

$<0,001$

3737

490

0,358

300

3448

$<0,001$

3449

778

$<0,001$

*Síndrome comum aos sexos 


\section{DISCUSSÃO}

Os achados deste estudo mostram que a verruga genital foi a síndrome mais prevalente entre os portadores de DST atendidos na atenção primária. Pessoas jovens e que tiveram múltiplos parceiros sexuais nos últimos três meses foram as que apresentaram maior proporção de verrugas e úlceras genitais. É possível que a vulnerabilidade para contrair DST/HIV seja maior entre pessoas jovens, pois estudos mostram que estão iniciando a vida sexual cada vez mais cedo e tendo mais parceiros sexuais ${ }^{20,21}$.

De forma semelhante aos achados de outras pesquisas ${ }^{22,23}$, encontrou-se que pessoas com boa escolaridade (nove anos ou mais de estudo) apresentaram mais síndromes genitais, situação que, especificamente neste estudo, pode estar relacionada ao perfil dos usuários que buscam essa unidade, considerando que ela se encontra num bairro nobre de Fortaleza, próximo à beira-mar, com grande fluxo de turismo e onde residem pessoas de classe média alta.

Acredita-se que a associação do resultado reagente do exame de VDRL em pessoas com úlcera genital possa não ser necessariamente devido ao cancro duro, manifestação da fase primária da sífilis. $O$ fato de não causar dor e de ser autolimitado, além da dificuldade de acesso aos serviços de saúde, pode fazer com que as pessoas com cancro dificilmente procurem os serviços ainda na fase inicial da infecção. Ademais, estudo realizado em pacientes com úlcera genital em clínicas de DST em Manaus encontrou uma predominância do vírus herpes simples como causador de úlcera genital ${ }^{24}$.

As pessoas com verruga apresentaram resultado reagente para o HIV. É comum a presença de mais de uma DST, visto que há associação entre elas e o $\mathrm{HIV}^{25}$. Ademais, um estudo de metaanálise encontrou associação entre a presença do papilomavírus humano e infecção pelo HIV ${ }^{26}$.

Observou-se que as mulheres apresentaram mais úlcera, verruga, mais de uma síndrome genital no momento da consulta e realizaram mais o VDRL e o anti-HIV que os homens. Mulheres são mais propensas a ter sintomatologia devido à anatomia da genitália feminina e, por outro lado, o fato de não terem abertura para conversar com o parceiro, não conseguindo conciliar o uso do preservativo, e de não se perceberem como em risco às DST pode aumentar sua vulnerabilidade ${ }^{27}$.

Ademais, a anatomia feminina propicia que as mulheres apresentem sintomatologia com maior frequência que os homens.

Neste estudo, os homens apresentaram mais resultados reagentes para o HIV, corroborando dados epidemiológicos nacionais de $\mathrm{Aids}^{28}$. Essa situação pode estar relacionada às questões comportamentais, uma vez que estudos mostram ser comum entre os homens manter práticas sexuais com vários(as) parceiros(as), simultaneamente ${ }^{29,30}$.

Uma limitação deste estudo éa ausência de maiores informações acerca do comportamento sexual, como início de vida sexual, práticas e orientação sexual, o que compromete uma análise mais detalhada de variáveis que possam estar relacionadas à presença de DST/HIV.

Chama atenção a diferença na aceitação do exame de VDRL quando comparado com o anti-HIV, tanto entre homens quanto entre mulheres. É possível que a maior recusa pelo anti-HIV tenha ocorrido em função do medo do diagnóstico reagente, situação que normalmente não ocorre em relação ao exame de VDRL. Durante o aconselhamento, deve ocorrer uma avaliação da vulnerabilidade e risco para essas infecções e é provável que portadores de DST tenham reconhecido uma maior exposição ao vírus HIV, o que pode ter contribuído para a recusa do teste. Estudo com amostra representativa da população brasileira realizado nos anos de 1998 e 2005 mostrou que pessoas que se autoavaliam em risco recusam mais o teste anti-HIV ${ }^{31}$.

É possível também que as pessoas aceitem o exame de VDRL devido à falta de conhecimento acerca das implicações provocadas pela infecção, visto que os serviços costumam dar maior prioridade ao aconselhamento para HIV/Aids em detrimento das outras DST. São muitos os estímulos por meio de campanhas educativas ${ }^{32}$ para que as pessoas realizem o teste anti-HIV. Pesquisa realizada com pessoas sexualmente ativas em São Paulo e Recife encontrou que todos os entrevistados revelaram maior conhecimento a respeito da Aids do que em relação às outras DST ${ }^{33}$.

Por outro lado, houve uma aceitação geral para ambos os testes, o que deve estar relacionado à própria organização interna da unidade, que favorece que as pessoas atendam as recomendações para o manejo dos casos de DST e a oferta dos exames VDRL e anti-HIV. A coleta do material biológico acontece logo após o atendimento, não sendo necessário o paciente retornar em outro dia.

Há também o fato de a maioria dos casos analisados neste estudo ter sido de pessoas com boa escolaridade, o que pode facilitar a compreensão e o reconhecimento da exposição aos fatores de risco e favorecer que as pessoas aceitem mais os testes.

\section{CONCLUSÃO}

Pode-se concluir que as pessoas com DST atendidas na unidade primária de saúde de Fortaleza são pessoas jovens com boa escolaridade e múltiplos parceiros sexuais. As mulheres apresentaram mais síndromes genitais e aceitaram realizar mais os exames de VDRL e anti-HIV. A infecção pelo HIV foi mais prevalente entre os homens.

Homens e mulheres apresentam especificidades comportamentais que exigem a elaboração de estratégias diferenciadas de prevenção. Essas estratégias devem considerar as questões de gênero que tornam os comportamentos diferenciados entre os sexos e podem aumentar a vulnerabilidade às DST e ao HIV.

A análise dos casos de síndromes genitais atendidos na atenção primária pode contribuir na elaboração de políticas de saúde direcionadas para prevenção de DST/Aids na população. 


\section{REFERÊNCIAS}

1. World Health Organization. Sexually transmitted infections. Fact sheet $\mathrm{N}^{\circ} 110$ [Internet]. 2011 [citado em 2014 nov 20]. Disponível em: http:// www.who.int/mediacentre/factsheets/fs110/en/index.html

2. Brasil. Ministério da Saúde. Manual de controle das doenças sexualmente transmissíveis. DST. 4a ed. Brasília: Ministério da Saúde; 2006. (Série Manuais, no. 68)

3. World Health Organization. Prevalence and incidence of selected sexually transmitted infections. Chlamydia, Neisseria gonorrhoeae, syphilis and Trichomonas vaginalis. Geneva: World Health Organization; 2011.

4. Gaydos CA. STI management and control in North America IUSTI region Sex Transm Infect. 2011;87(Supl 2):ii2-6. http://dx.doi.org/10.1136/ sextrans-2011-050287. PMid:22110147.

5. Macaluso M, Wright-Schnapp TJ, Chandra A, Johnson R, Satterwhite CL, Pulver A, et al. A public health focus on infertility prevention, detection, and management. Fertil Steril. 2010;93(1):16.e1-10. http://dx.doi.org/10.1016/j. fertnstert.2008.09.046. PMid:18992879.

6. Rosa MI, Medeiros LR, Rosa DD, Bozzeti MC, Silva FR, Silva BR. Papilomavírus humano e neoplasia cervical. Cad Saúde Pública. 2009;25(5):953-64. PMid:19488480.

7. Guimarães AGDP, Araújo JR, Andrade RV, Costa CM, Galvão RS, Hada $\mathrm{AL}$, et al. Coinfection of Epstein-Barr virus, cytomegalovirus, herpes simplex virus, human papillomavirus and anal intraepithelial neoplasia in HIV patients in Amazon, Brazil. J Coloproctol (Rio J.). 2012;32(1):18-25.

8. Reis AAS, Paula LB, Paula AAP, Saddi VA, Cruz AD. Aspesctos clínicoepidemiológicos associados ao câncer de pênis. Cien Saúde Colet. 2010;15(Supl 1):1105-11. http://dx.doi.org/10.1590/S1413-81232010000700018. PMid:20640268.

9. Todd J, Grosskurth H, Changalucha J, Obasi A, Mosha F, Balira R, et al. Risk factors influencing HIV infection incidence in a rural African population: a nested case-control study. J Infect Dis. 2006;193(3):458-66. http://dx.doi. org/10.1086/499313. PMid:16388496.

10. Brasil. Ministério da Saúde. Prevalências e freqüências relativas de Doenças Sexualmente Transmissíveis em populações selecionadas de seis capitais brasileiras. Brasília: Ministério da Saúde; 2008.

11. Lima LH, Viana MC. Prevalência e fatores de risco para infecção por HIV, sifilis, hepatite B, hepatite C e HTLV-I/II em parturientes e gestantes de baixa renda atendidas na Região Metropolitana de Vitória, Espírito Santo, Brasil. Cad Saúde Pública. 2009;25(3):668-76. http://dx.doi.org/10.1590/ S0102-311X2009000300021. PMid:19300855.

12. Machado Fo AC, Sardinha JFJ, Ponte RL, Costa EP, Silva SS, Martinez-Espinosa FE. Prevalência de infecção por HIV, HTLV, VHB e de sífilis e clamídia em gestantes numa unidade de saúde terciária na Amazônia ocidental brasileira. Rev Bras Ginecol Obstet. 2010;32(4):176-83. PMid:20625686.

13. Mukenge-Tshibaka L, Alary M, Lowndes CM, Van Dyck E, Guédou A, Geraldo N, et al. Syndromic vesus laboratory-based diagnosis of cervical infections among female sex works in Beni. Sex Transm Dis. 2002;29(6):32430. http://dx.doi.org/10.1097/00007435-200206000-00003. PMid:12035021.

14. Garcia PJ, Benzaken AS, Galban E, ALAC-ITS members. STI management and control in Latin America: where do we stand and where do we go from here? Sex Transm Infect. 2011;87(Supl 2):ii7-9. http://dx.doi.org/10.1136/ sextrans-2011-050177. PMid:22110156.

15. Araújo MAL, Leitão GCM. Acesso à consulta a portadores de doenças sexualmente transmissíveis: experiências de homens em uma unidade de saúde de Fortaleza, Ceará, Brasil. Cad Saúde Pública. 2005;21(2):396-403. http://dx.doi.org/10.1590/S0102-311X2005000200006. PMid:15905902.

16. Naves JOS, Castro LLC, Melo GF, Giavoni A, Merchán-Hamann E. Práticas de atendimento a DST nas farmácias do Distrito Federal, Brasil: um estudo de intervenção. Cad Saúde Pública. 2008;24(3):577-86. http://dx.doi. org/10.1590/S0102-311X2008000300011. PMid:18327445.

17. Domingues RMSM, Lauria LM, Saraceni V, Leal MC. Manejo da sífilis na gestação: conhecimentos, práticas e atitudes dos profissionais prénatalistas da rede SUS do município do Rio de Janeiro. Cien Saúde Colet. 2013;18(5):1341-51. http://dx.doi.org/10.1590/S1413-81232013000500019. PMid:23670462.

18. Araújo MAL, Silva DMA, Silva RM. Gonçalves MLC. Análise da qualidade dos registros nos prontuários de gestantes com exame de VDRL reagente. Rev APS. 2008;11(1):4-9.

19. World Health Organization. Guidelines for the management of sexually transmitted infections. Geneva: WHO; 2001.

20. Parra Villarroel J, Domínguez Placencia J, Maturana Rosales J, Pérez Villegas $\mathrm{R}$, Carrasco Portiño M. Conocimiento y percepción de adolescentes sobre el servicio de planificación familiar en Chile. Salud Colect. 2013;9(3):391400. http://dx.doi.org/10.18294/sc.2013.193. PMid:24500551.

21. Muñoz MAG, Nitschke RG, Tholl AD. Sexual behavior in the everyday life of adolescents and young adults from the hip hop culture. Texto contexto - enferm. 2014;23(1):126-33.

22. Fagundes LJ, Vieira Jr EE, Moyses ACMC, Lima FD, Morais FRB, Vizinho NL. Sexually transmitted diseases in a specialized STD healthcare center: epidemiology and demographic profile from january 1999 to december 2009. An Bras Dermatol. 2013;88(4):523-9. http://dx.doi.org/10.1590/ abd1806-4841.20132149. PMid:24068122.

23. Pedrosa VL, Galban E, Benzaken A, Vasquez FG, Izan Jr JL. DST e suas determinantes: quatro anos de vigilância em um centro sentinela no Estado do Amazonas - Brasil. DST - J bras Doenças Sex Transm. 2011;23(2):57-65.

24. Naveca FG, Sabidó M, Almeida TAP, Veras EA, Mejía MCC, Galban E, et al. Etiology of genital ulcer disease in a sexually transmitted infection reference center in Manaus, Brazilian Amazon. PLoS One. 2013;8(5):e63953. http:// dx.doi.org/10.1371/journal.pone.0063953. PMid:23704961.

25. Mhlongo S, Magooa P, Müller EE, Nel N, Radebe F, Wasserman E, et al. Etiology and STI/HIV coinfections among patientes with urethral and vaginal discharge syndromes in South Africa. Sex Transm Dis. 2010;37(9):566-70. http://dx.doi.org/10.1097/OLQ.0b013e3181d877b7. PMid:20502394.

26. Houlihan CF, Larke NL, Watson-Jones D, Smith-McCune KK, Shiboski S, Gravitt PE, et al. Human papillomavirus infection and increased risk of HIV acquisition. A systematic review and meta-analysis. AIDS. 2012;26(17):221122. http://dx.doi.org/10.1097/QAD.0b013e328358d908. PMid:22874522.

27. Guanilo MCTU, Takahashi RFT, Bertolozzi MR. Avaliação da vulnerabilidade de mulheres às Doenças Sexualmente Transmissíveis - DST e ao HIV: construção e validação de marcadores. Rev Esc Enferm USP. 2014;48(N. esp):156-63.

28. Boletim Epidemiológico HIV AIDS. Brasília: Ministério da Saúde. Ano 3 , No. 1, 2014.

29. Scanavino M, Abdo CHN. Parceiros sexuais nos últimos 12 meses e parceiros significativos ao longo da vida, segundo o Estudo da Vida Sexual do Brasileiro. Diagn Tratamento. 2010;15(3):138-42. 
30. Figueiredo WS, Schraiber LB. Concepções de gênero de homens usuários e profissionais de saúde de serviços de atenção primária e os possíveis impactos na saúde da população masculina, São Paulo, Brasil. Cien Saúde Colet. 2011;16(Supl 1):935-44. http://dx.doi.org/10.1590/S141381232011000700025. PMid:21503441.

31. França Junior I, Calazans G, Zucchi EM, Grupo de Estudos em População, Sexualidade e Aids. Mudanças no âmbito da testagem anti-HIV no Brasil entre 1998 e 2005. Rev Saude Publica. 2008;42(Supl 1):84-97. http://dx.doi. org/10.1590/S0034-89102008000800011. PMid:18660928.
32. Brasil. Ministério da Saúde. Departamento de DST, Aids e Hepatites Virais. Campanhas [Internet]. 2012 [citado em 2012 jan 02]. Disponível em: http:// www.aids.gov.br/campanhas

33. Garcia S, Souza FM. Vulnerabilidades ao HIV/aids no Contexto Brasileiro: iniquidades de gênero, raça e geração. Saúde soc. 2010;19(Supl 2):9-20.

Recebido em: Mar. 26, 2015 Aceito em: Out. 27, 2015 